\title{
A Lattice-Boltzmann model for low-Mach reactive flows
}

\author{
Y. Feng ${ }^{\mathrm{a}}$, M. Tayyab ${ }^{\mathrm{a}}$, P. Boivin ${ }^{1 \mathrm{a}}$ \\ ${ }^{a}$ Aix Marseille Univ, CNRS, Centrale Marseille, M2P2, Marseille, France
}

\begin{abstract}
A new Lattice-Boltzmann model for low-Mach reactive flows is presented. Based on standard lattices, the model is easy to implement, and is the first, to the authors' knowledge, to pass the classical freely propagating flame test case as well as the counterflow diffusion flame, with strains up to extinction. For this presentation, simplified transport properties are considered, each species being assigned a separate Lewis number. In addition, the gas mixture is assumed to be calorically perfect. Comparisons with reference solutions show excellent agreement for mass fraction profiles, flame speed in premixed mixtures, as well as maximum temperature dependence with strain rate in counterflow diffusion flames.
\end{abstract}

Keywords: lattice Boltzmann; reacting flows; combustion

\section{Introduction}

Industries from the aerospace, aeronautic and automotive sectors are increasingly relying on numerical simulation tools. From the occasional use of a research and development department, these tools progressively made

\footnotetext{
${ }^{1}$ Corresponding author: pierre.boivin@univ-amu.fr
} 
it to conception and production departments, where they help to continuously improve designs. The field of low-Mach external aerodynamics and aeroacoustics, have been particularly impacted by the rapid development of Lattice-Boltzmann (LB) methods [1] in the last five to ten years. From industrial benchmarks [2 4], these methods quickly ramped up to full scale applications: full-scale cars [5, 6], full-scale aircraft engines [7] and even fullscale aircrafts [8, 9], oftentimes with outstanding results.

Combustion modelling in the LB framework, however, remain relatively marginal within the combustion community. A hybrid finite difference lattice Boltzmann model has been presented for the simulation of low Mach number flows with significant density changes by Filippova et al [10] almost twenty years ago. An LB model for combustion modelling was then presented by Yamamoto and coworkers [11 13] for steady and unsteady reactive flows. About ten years ago, a pressure-based lattice Boltzmann model was presented by Chen et al. for low Mach number combustion simulations by [14, 15], based on an incompressible LB model developed earlier by Guo et al [16]. An entropic lattice Boltzmann model on two-dimensional standard lattice was also presented for compressible thermal flows and extended to combustion applications [17, 18]. More recently, detailed kinetics were successfully implemented in the LB framework [19, 20].

Thermodynamic closure is one of the key issues in extending the LB capabilities from low-Mach aerodynamics and aero-acoustics to reactive flows, and obtaining satisfactory results in cases with significant thermal expansion is challenging, as indicated by the low-number of combustion models in the LB context. Most of LB models mentioned above for reactive flows were 
specifically applied under constant pressure, and lack full coupling between thermodynamics and the flow. In particular, because their equilibrium density distribution function only depends on the local density, hydrodynamic pressure and velocity, and not on temperature, these models may fail at setting in motion fluids at rest when heat-release is applied. This aspect is however critical in representing canonical combustion phenomena, such as ignition in a mixing layer, or the dynamics of freely propagating flame in a premixed mixture.

To address this issue, more complex, multi-speed models with aid of finite volume approach were developed for subsonic and supersonic flows [21], including detonations. Alternatively, coupled lattice Boltzmann models on standard lattices have been investigated in low Mach thermal compressible flows [22, 23].

The model presented in this work is based on the latter [23] and takes full advantage of the simplicity and practicality of standard lattice models. It is able to deal with multi-component, calorically perfect gas mixtures, and the coupling between the thermodynamics and the flow is two-way. Simplified transport properties are considered, with non-unity Lewis number set for each component. This new LB model for low-Mach reactive flows is presented in the first Section. The second Section validates the model using Cantera [24] as a reference on canonical combustion test cases of premixed and nonpremixed combustion. 


\section{Lattice-Boltzmann combustion modelling}

Introductory comments: athermal LB method

Lattice Boltzmann methods intend to solve the continuous Boltzmann equation through discretization of space, time, and velocity [1, 25, 26]. In the so-called $D n Q m$ model, a $n$-dimensional physical space is filled with a regular lattice (or a Cartesian grid) and velocity space is discretized on a set of lattice tensor $\boldsymbol{E}_{\boldsymbol{m}}^{\boldsymbol{n}}=\left[\boldsymbol{c}_{0}, \ldots, \boldsymbol{c}_{i}, \ldots, \boldsymbol{c}_{m-1}\right]$. On every lattice node $\boldsymbol{x}, f_{i}(\boldsymbol{x}, t)$ denotes the density distribution of particle with velocity $\boldsymbol{c}_{i}$, thus the local density $\rho$ and momentum $\rho \boldsymbol{u}$ are defined as $\rho=\Sigma_{i} f_{i}$ and $\rho \boldsymbol{u}=\Sigma_{i} \boldsymbol{c}_{\boldsymbol{i}} f_{i}$.

A single time relaxation process is used to model the collision term of Boltzmann equation in this study, which is the so-called Bhatnagar-GrossKrook (BGK) model [27, 28].

$$
f_{i}(\boldsymbol{x}+\boldsymbol{c} \delta t, t+\delta t)-f_{i}(\boldsymbol{x}, t)=-\frac{1}{\tau}\left[f_{i}(\boldsymbol{x}, t)-f_{i}^{e q}(\boldsymbol{x}, t)\right]
$$

where $\tau$ is the relaxation parameter, $\delta t$ is the time increment and $\delta t=\delta \boldsymbol{x} / \boldsymbol{c}$. $f_{i}(\boldsymbol{x}, t), f_{i}(\boldsymbol{x}+\boldsymbol{c} \delta t, t+\delta t)$ are the distribution functions associated with the $i^{\text {th }}$ discrete velocity $\boldsymbol{c}_{i}$, and $f_{i}^{e q}$ is the $i^{\text {th }}$ equilibrium distribution function given by [1, 25]

$$
f_{i}^{e q}=\rho w_{i}\left[1+\frac{\boldsymbol{c}_{i} \cdot \boldsymbol{u}}{c_{s}^{2}}+\frac{\left(\boldsymbol{c}_{i} \cdot \boldsymbol{u}\right)^{2}}{2 c_{s}^{4}}-\frac{\boldsymbol{u}^{2}}{2 c_{s}^{2}}\right]
$$

where $w_{i}$ is the weight coefficient associated to discrete velocity $\boldsymbol{c}_{i}$ and $c_{s}$ is sound speed.

Using the Chapman-Enskog multiscale technique [28], the equivalent NavierStokes equations can be recovered as

$$
\frac{\partial \rho}{\partial t}+\nabla \cdot(\rho \boldsymbol{u})=0
$$




$$
\frac{\partial \rho \boldsymbol{u}}{\partial t}+\nabla \cdot(\rho \boldsymbol{u} \boldsymbol{u})=-\nabla p+\nabla \cdot\left[\rho \nu\left(\nabla \boldsymbol{u}+(\nabla \boldsymbol{u})^{T}\right)\right]
$$

where the kinematic viscosity $\nu$ is related to the relaxation parameter through

$$
\tau=\frac{\nu}{c_{s}^{2} \delta t}+\frac{1}{2}
$$

And, the pressure is related to the density by the equation of the state $p=$ $\rho c_{s}^{2}$. The equation of state used in this base model is clearly not suitable for reactive flows, as no temperature is considered. The most simple acceptable equation of state for reactive flows is probably the ideal gas law, relating the gas pressure and temperature through

$$
p=\rho \bar{r} T
$$

where $\bar{r}=R / \bar{W}$, with $\mathrm{R}$ the gas constant, and

$$
1 / \bar{W}=\sum_{k} Y_{k} / W_{k}
$$

linking the mean molecular weight $\bar{W}$ to the species molecular weights $W_{k}$ and the species mass fractions $Y_{k}$.

\section{A hybrid thermal LB model}

Considering the third order Hermite expansion of Maxwell-Boltzmann distribution, one can obtain the equilibrium distribution function in discrete Gauss-Hermite space [26]

$$
\begin{aligned}
& f_{i}^{e q}=f_{i}^{(0)}=\rho w_{i}\left[1+\frac{c_{i \alpha} u_{\alpha}}{c_{s}^{2}}+\frac{A_{\alpha \beta}^{(2)} Q_{i \alpha \beta}^{(2)}}{2 c_{s}^{4}}+\frac{A_{\alpha \beta \gamma}^{(3)} Q_{i \alpha \beta \gamma}^{(3)}}{6 c_{s}^{6}}\right], \\
& A_{\alpha \beta}^{(2)}=u_{\alpha} u_{\beta}+(\theta-1) c_{s}^{2} \delta_{\alpha \beta}, \quad Q_{i \alpha \beta}^{(2)}=c_{i \alpha} c_{i \beta}-c_{s}^{2} \delta_{\alpha \beta}, \\
& A_{\alpha \beta}^{(3)}=u_{\alpha} u_{\beta} u_{\gamma}+(\theta-1) c_{s}^{2}[u \delta]_{\alpha \beta \gamma}, \quad Q_{i \alpha \beta}^{(3)}=c_{i \alpha} c_{i \beta} c_{i \gamma}-c_{s}^{2}[c \delta]_{\alpha \beta \gamma},
\end{aligned}
$$


where $[c \delta]_{\alpha \beta \gamma}=c_{\alpha} \delta_{\beta \gamma}+c_{\beta} \delta_{\alpha \gamma}+c_{\gamma} \delta_{\alpha \beta}, \delta_{\alpha \beta}$ is the Kronecker symbol and $\theta$ is the non-dimensional temperature

$$
\theta=\frac{\bar{r} T}{c_{s}^{2}}=\frac{R T}{c_{s}^{2}} \sum_{k} \frac{Y_{k}}{W_{k}}
$$

The moment of equilibrium distribution function on the nearest neighbor type lattices, hereafter referred to as standard lattices (D2Q9, D3Q19, D3Q27, etc.), are then

$$
\begin{gathered}
\sum_{i} f_{i}^{(0)}=\rho \\
\sum_{i} f_{i}^{(0)} c_{i \alpha}=\rho u_{\alpha}, \\
\sum_{i} f_{i}^{(0)} c_{i \alpha} c_{i \beta}=\rho \bar{r} T \delta_{\alpha \beta}+\rho u_{\alpha} u_{\beta}, \\
\sum_{i} f_{i}^{(0)} c_{i \alpha} c_{i \beta} c_{i \gamma}=\rho \bar{r} T\left(u_{\alpha} \delta_{\beta \gamma}+u_{\beta} \delta_{\gamma \alpha}+u_{\gamma} \delta_{\beta \alpha}\right)+\rho u_{\alpha} u_{\beta} u_{\gamma}+\Psi_{\alpha \beta \gamma},
\end{gathered}
$$

where $\Psi_{\alpha \beta \gamma}$ is a deviation term due to defect of symmetry of standard lattices for the third order moment. This deviation can be corrected by introduction of an extra force term $s_{i}=Q_{i \alpha \beta} \frac{\partial}{\partial x_{\gamma}} \Psi_{\alpha \beta \gamma}$ in the lattice Boltzmann equation. The LB equation with a force term in the second order accuracy scheme is given as [29]

$$
\begin{gathered}
f_{i}(\boldsymbol{x}+\boldsymbol{c} \delta t, t+\delta t)=f_{i}(\boldsymbol{x}, t)- \\
-\frac{1}{\tau}\left[f_{i}(\boldsymbol{x}, t)-f_{i}^{e q}(\boldsymbol{x}, t)\right] \\
+\left(1-\frac{1}{2 \tau}\right) s_{i}
\end{gathered}
$$

where the external force term $s_{i}$ reads [23]

$$
s_{i}=Q_{i x x} \frac{\partial}{\partial x}\left[\rho u_{x}\left(1-\theta-u_{x}^{2}\right)\right]+Q_{i y y} \frac{\partial}{\partial y}\left[\rho u_{y}\left(1-\theta-u_{y}^{2}\right)\right]
$$


in two dimensions.

The mass and momentum conservation equations derived through ChapmanEnskog expansion from this model remain unchanged compared to the classical athermal version (3) and (44), with the exception of the equation of state and the relation between the relaxation time and the viscosity (5), which now reads

$$
\tau=\frac{\nu}{\theta c_{s}^{2} \delta t}+\frac{1}{2}
$$

The approach proposed here is hybrid: coupled with this lattice Boltzmann description, temperature $T$ and mass fractions $Y_{k}$ equations are solved following a classical finite difference method

$$
\begin{aligned}
& \frac{\partial T}{\partial t}+u_{\alpha} \frac{\partial}{\partial x_{\alpha}} T=\frac{1}{\rho} \frac{\partial}{\partial x_{\alpha}}\left(\rho D_{T} \frac{\partial T}{\partial x_{\alpha}}\right)+\frac{\omega_{h}}{\rho c_{p}} \\
& \frac{\partial Y_{k}}{\partial t}+u_{\alpha} \frac{\partial}{\partial x_{\alpha}} Y_{k}=\frac{1}{\rho} \frac{\partial}{\partial x_{\alpha}}\left(\rho D_{k} \frac{\partial Y_{k}}{\partial x_{\alpha}}\right)+\frac{\omega_{k}}{\rho}
\end{aligned}
$$

$D_{k}$ and $D_{T}$ are respectively the $k^{t h}$ species and thermal diffusivities.

As to simplify the introduction of this model, we assume calorically gases, with a constant mixture heat capacity. Transport properties are specified through constant Prandtl number and constant Schmidt number for each species following [30]

$$
D_{T}=\frac{\nu}{P r}, \quad D_{k}=\frac{\nu}{S c_{k}}
$$

Physical and lattice units for length, time, mass and temperature are related through a reference length scale $L_{0}$, the physical sound speed $c_{s p}$ for space and time, a reference density $\rho_{0}$ for mass, and a reference temperature 
$T_{0}$. Accordingly, the physical variables can be converted from the quantities in lattice units ( $l$ subscript) as

$$
\begin{aligned}
& \delta x=L_{0} / N_{i}, \delta t=\delta x \cdot \frac{c_{s}}{c_{s p}}, \\
& t=N_{t} \cdot \delta t, u=u_{l} \cdot \frac{\delta x}{\delta t}, \\
& \mu=\mu_{l} \frac{\rho}{\rho_{l}} \frac{\delta x^{2}}{\delta t}
\end{aligned}
$$

where $c_{s p}$ is the reference physical sound speed. It could be set to the fresh gases sound speed, but in low-Mach flows, it is more convenient to keep it as a free parameter. This allows to freely accelerate the convergence rate, by varying the time step somewhere between a velocity based time-step (as in low-Mach solvers) and a sound-speed based time-step (as in compressible solvers). We recommend to adopt a high reference temperature (here $T_{0}=$ $3000 \mathrm{~K}$ ) as to restrict the variations of $\theta-1$ (let us remind that $\theta=\bar{r} T / c_{s}^{2}$ ) and improve numerical stability [31, 32].

\section{Model implementation}

A strong advantage of the present model is its simplicity. The algorithm we implemented is illustrated in Fig. 1. In essence, two solvers are simultaneously running:

- the Lattice Boltzmann (LB) solver, for mass and momentum conservation Eqs (3) and (4)

- the Finite Differences (FD) solver, for temperature $T$ and mass fractions $Y_{k}$ evolution through Eqs (16) and (17) 


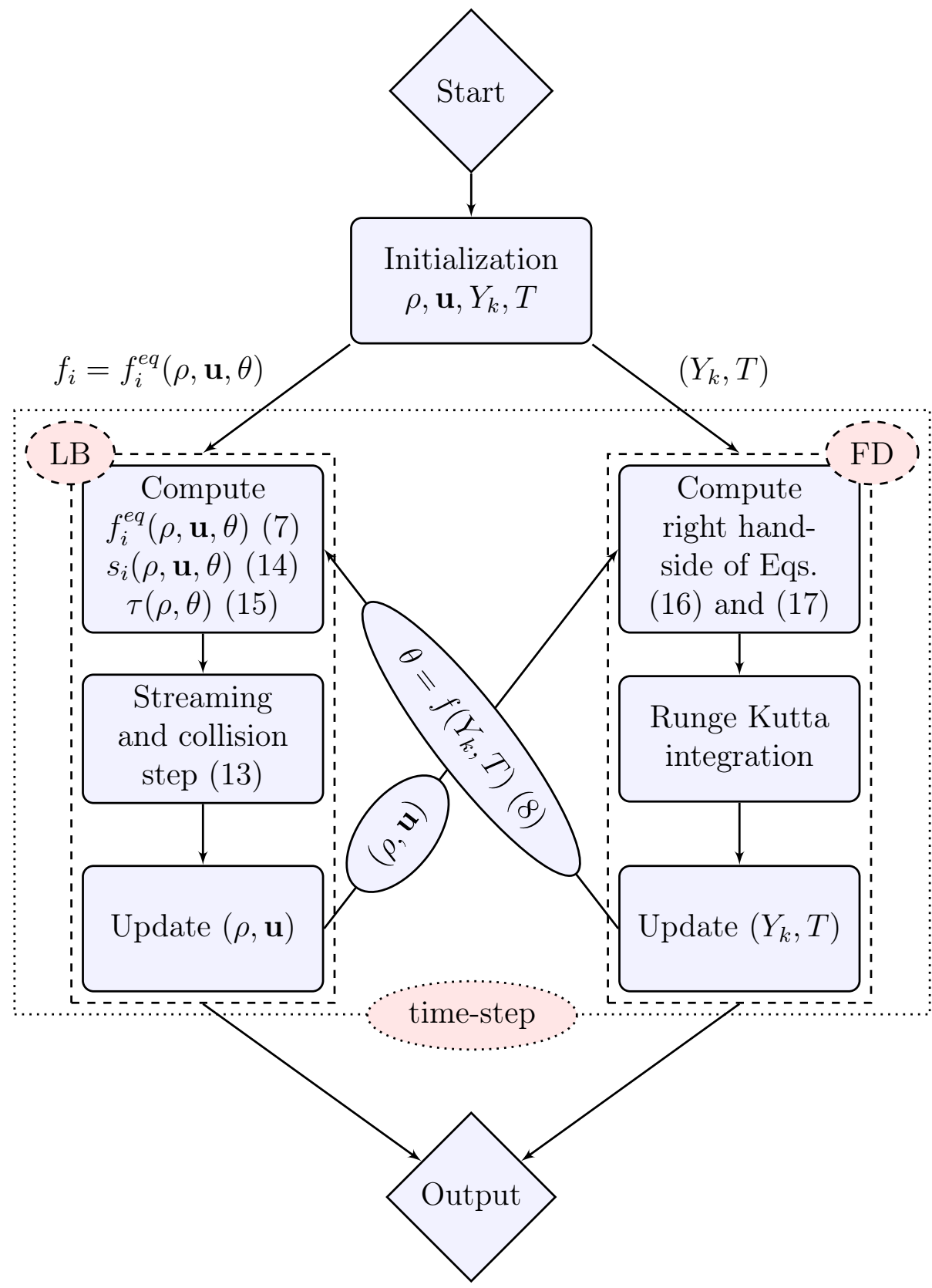

Figure 1: The algorithm proposed consists of a Lattice-Boltzmann (LB) solver coupled with a classical Finite Differences (FD) solver. Data exchanges between the two solvers are clearly identified. 
At the end of each time-step, the two solvers exchange macroscopic values: $(\rho, \mathbf{u})$ is sent from the LB to the FD solver, and the gas composition and temperature $\left(T, Y_{k}\right)$ from the FD to the LB solver.

The LB solver, being of very classical form as well as built on standard lattices, can share most of the features of standard LB methods. In particular, a regularized BGK collision model was employed to enhance the robustness [33, 34], albeit not strictly necessary for the test cases proposed here. The gradients in the correction term (14) are numerically implemented by an isotropic second order central difference scheme [35, 36].

The FD solver's implementation is also very straight-forward. Equations (16) and (17) are integrated through a second-order Runge-Kutta scheme in time and the same isotropic finite difference scheme was adopted for the convective and diffusive terms [35, 36].

Boundary conditions

A non-equilibrium bounce back scheme with a regularization procedure [37] is employed at the inlets for the LB solver, allowing to set all velocity components and the pressure (or density). ( $\left.T, Y_{k}\right)$ are hard-coded at inlets for the FD solver.

Outlets are treated as zero-gradient boundaries on microscopic distribution functions $f_{i}$ as well as $T$ and $Y_{k}$ variables.

\section{Validation through canonical combustion problems}

This section aims at validating the combustion model developed in a D2Q9 Lattice-Boltzmann framework against solutions from Cantera [24], a well-validated open source tool designed to compute quickly and efficiently. 
It incorporates and executes fundamental thermodynamics, chemical kinetics and species transport calculations.

\subsection{Premixed combustion: freely propagating flame}

Let us consider a multi-component gas consisting of $\mathrm{C}_{3} \mathrm{H}_{8}, \mathrm{O}_{2}, \mathrm{H}_{2} \mathrm{O}, \mathrm{CO}_{2}, \mathrm{~N}_{2}$, with Schmidt numbers listed in Tab. 1, along with the parameters from the viscosity's power-law,

$$
\mu=\mu_{\oslash}\left(\frac{T}{T_{\oslash}}\right)^{\beta},
$$

and the Prandtl number, defining now entirely the species and thermal diffuses properties through (18). In the above $T_{\oslash}=298 \mathrm{~K}$ is the reference temperature of the viscosity power-law, not to be confused with the LB reference temperature (3000K here). The mixture's heat capacity is assumed

$\begin{array}{cl}\mu_{\varnothing} & 1.782 \times 10^{-5} \\ \beta & 0.690 \\ P r & 0.682 \\ S_{\mathrm{C}_{3} \mathrm{H}_{8}} & 1.241 \\ S_{\mathrm{O}_{2}} & 0.728 \\ S_{\mathrm{CO}_{2}} & 0.941 \\ S_{\mathrm{H}_{2} \mathrm{O}} & 0.537 \\ S_{\mathrm{N}_{2}} & 0.690\end{array}$

Table 1: Power-law's viscosity coefficients (SI units), Prandtl number and Schmidt numbers for each species.

to be constant $C_{p}=1008 \mathrm{~J} / \mathrm{K} / \mathrm{kg}$.

Last but not least, a one-step Arrhenius kinetic model is introduced, following global reaction $\mathrm{C}_{3} \mathrm{H}_{8}+5 \mathrm{O}_{2} \longrightarrow 3 \mathrm{CO}_{2}+4 \mathrm{H}_{2} \mathrm{O}$, associated with the kinetic rate $\omega=k \cdot C_{\mathrm{C}_{3} \mathrm{H}_{8}} \cdot C_{\mathrm{O}_{2}} \cdot e^{-E_{a} / R T}$, where $k=9.9 \times 10^{13} \mathrm{~cm}^{3} \mathrm{~mol}^{-1} \mathrm{~s}^{-1}$, $E_{a}=30 \mathrm{kcal} / \mathrm{mol}$, and $C_{i}$ are the molar concentrations for species $i$. 
Having introduced these kinetic, thermodynamic and transport properties, let us now consider the most popular laminar flame, the so-called freely propagating flame. A pseudo one-dimensional domain is now initialized as $\left[T, p, u, Y_{\mathrm{C}_{3} \mathrm{H}_{8}}, Y_{\mathrm{O}_{2}}, Y_{\mathrm{CO}_{2}}, Y_{\mathrm{H}_{2} \mathrm{O}}\right]=\left\{\begin{array}{ll}{[300 \mathrm{~K}, 1 \mathrm{~atm}, 0,0.06,0.21,0,0],} & \text { if } x<L / 2 \\ {[3070 \mathrm{~K}, 1 \mathrm{~atm}, 0,0,0,0.18,0.1],} & \text { else. }\end{array}\right.$,

the fresh gases corresponding to a stoichiometric mixture of propane and air in ambient conditions (1atm., 300K), and the burnt gases being set to the adiabatic temperature at ambient pressure. The right side consists of the combustion products of the fresh gases combustion, with $3070 \mathrm{~K}$ being the adiabatic flame temperature. Let us remind here that we are considering constant $C_{p}$ and a reduced number of species, which both contribute to the rather high burnt gas temperature.

While Cantera [24] includes an straight-forward automated procedure for computing the flame speed, our LB model does not. Computations were initialized as a Heaviside function between the fresh and burnt states. The velocity $U_{f}$ is imposed at the inlet, with $U_{f}$ set arbitrarily, given the flame does not leave the computational domain before convergence.

After initial establishment of the flow, the flame speed can be estimated through

$$
S_{L}=U_{f}-\frac{\rho_{f} U_{f}-\rho_{b} U_{b}}{\rho_{f}-\rho_{b}},
$$

where the $f$ subscript indicate the fresh gases, and $b$ denotes the burnt gases properties (respectively the first and last points of the simulation domain).

Mass fractions and temperature profiles in the stoichiometric premixed 
flame are presented in Fig. 2, showing excellent agreement between our method and the Cantera-computed reference.

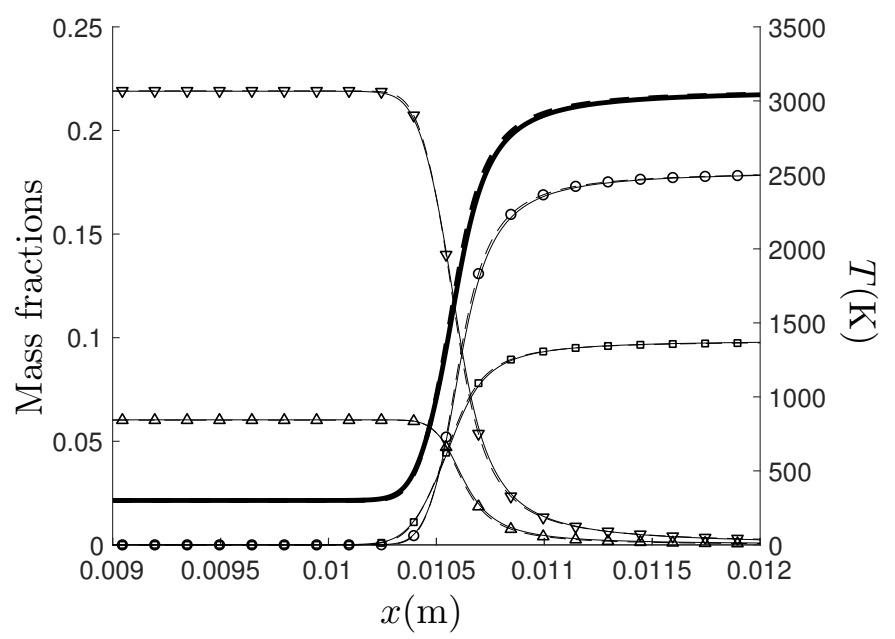

Figure 2: Freely propagating flame: Temperature profile (thick line), $\mathrm{C}_{3} \mathrm{H}_{8}(\Delta), \mathrm{O}_{2}(\nabla)$, $\mathrm{CO}_{2}(\circ)$ and $\mathrm{H}_{2} \mathrm{O}(\square)$ mass fractions. The fresh gases consist of a stoichiometric mixture of propane and air, at $300 \mathrm{~K}$ and atmospheric pressure. Cantera reference (plain line), and present model (dashed line).

As an additional validation, the equivalence ratio dependence of the flame speed, estimated through Eq. (22) is presented in Fig. 33 and compared with the Cantera reference value. The left side of the plot indicates convergence histories, through the time evolution of the velocity evaluated as (22). For most computations, $U_{f}=0$, but 0.4 and $0.8 \mathrm{~m} / \mathrm{s}$ were also tested at $\varphi=1$, as to check the consistence of the model. As indicated by the convergence histories, the obtained flame speed is independent of the inlet velocity $U_{f}$ and does indeed converge to Cantera's predictions. 


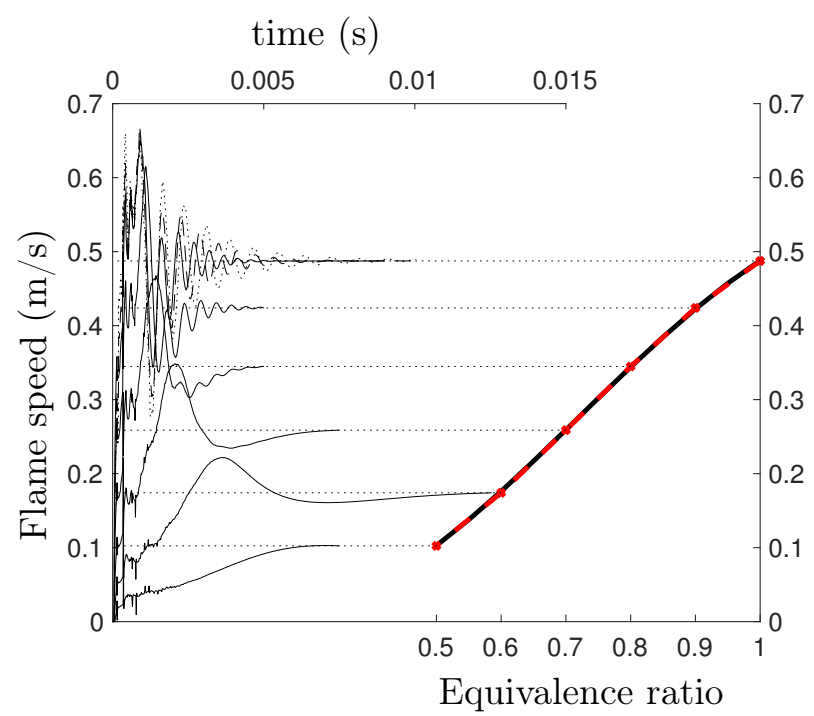

Figure 3: Freely propagating flame: Variation with equivalence ratio of the flame speed in propane/air mixtures at $300 \mathrm{~K}$ and atmospheric pressure. Cantera reference (plain dark line), and present model (dashed colored line). On the secondary x-axis are plotted the convergence histories for the LBM computed flame speed $S_{L}$ estimated through Eq. (22). $U_{f}=0$ for all $\varphi=0.5,0.6,0.7,0.8,0.9$ and 1 (thin lines). Dotted and dashed lines histories for $\varphi=1$ correspond to $U_{f}=0.4$ and 0.8 , respectively.

\subsection{Non-premixed combustion: counter-flow diffusion flame}

As a concluding validation, counter-flow diffusion flame is now considered. This test case, consisting of two counter flows (oxidizer and fuel), can in principle be solved as a 1D problem with an adequate choice of variables [38, 39], as done in Cantera [24]. For validation purposes, however, it is sometimes convenient to solve a $2 \mathrm{D}$ problem. A two-dimensional rectangular $L \times l$ is adopted as geometry domain for our LBM computation. Top and bottom boundaries are inlet, whereas the left boundary is a symmetry, and the right is a pressure outlet. Kinetic, thermodynamic and transport properties are identical to those of the premixed flame presented above.

The fuel mixture with uniform density $\rho_{f}$, speed $U_{f}$ and temperature $T_{f}=300 \mathrm{~K}$ is injected from the upper boundary at atmospheric pressure. 
On the opposite inlet, air is uniformly injected at $\rho_{o x}, U_{o x}, T_{o x}=300 \mathrm{~K}$ and atmospheric pressure. Fuel and oxidizer mixtures have the same $\mathrm{N}_{2}$ mass fraction $Y_{\mathrm{N}_{2}}=0.767$, the remainder being propane and dioxygen, respectively. The set up for $L / l=2$ is illustrated in Fig. 4 through the superposition of streamlines and temperature profiles, for mass flow rates $\rho_{f} U_{f}=\rho_{o x} U_{o x}=0.2 \mathrm{~kg} \mathrm{~m}^{2} \mathrm{~s}^{-1}$. The inter-nozzle distance is $L=20 \mathrm{~mm}$, corresponds to a strain of $16.5 \mathrm{~s}^{-1}$. It is interesting to note that no specific treatment was found necessary in the inlet/outlet corners, whereas with more conventional methods authors typically extend the domain as to have an outlet far from the inlet [40].

Mass fractions, axial velocity and temperature profiles are reported in Fig. 5, showing an outstanding agreement. The error in maximum temperature is less than $1 \mathrm{~K}$. Somewhat larger errors can be seen on the axial velocity profile, to be attributed to the fact that the two-dimensional setup is not strictly equivalent to the $1 \mathrm{D}$ problem solved in Cantera.

It becomes then of interest to check whether the dependence of the maximum temperature with the strain rate is correctly reproduced with the LB model. The $2 \mathrm{D}$ configuration leading to an unstable stagnation line when the domain is large, simulations were ran on a thinner domain, with $L / l=100$. Results, albeit slightly altered in accuracy remain close to the reference, with an error of $15 \mathrm{~K}$ in maximum temperature in the conditions of Fig. 5. The dependence of the maximum temperature with strain rate in this thin $2 \mathrm{D}$ domain are reported in Fig. 6, showing a very good agreement.

The $\times$ symbol at the lowest strain rate corresponds to the result on the large domain simulation $(L / l=2)$, showing the errors to come from the 


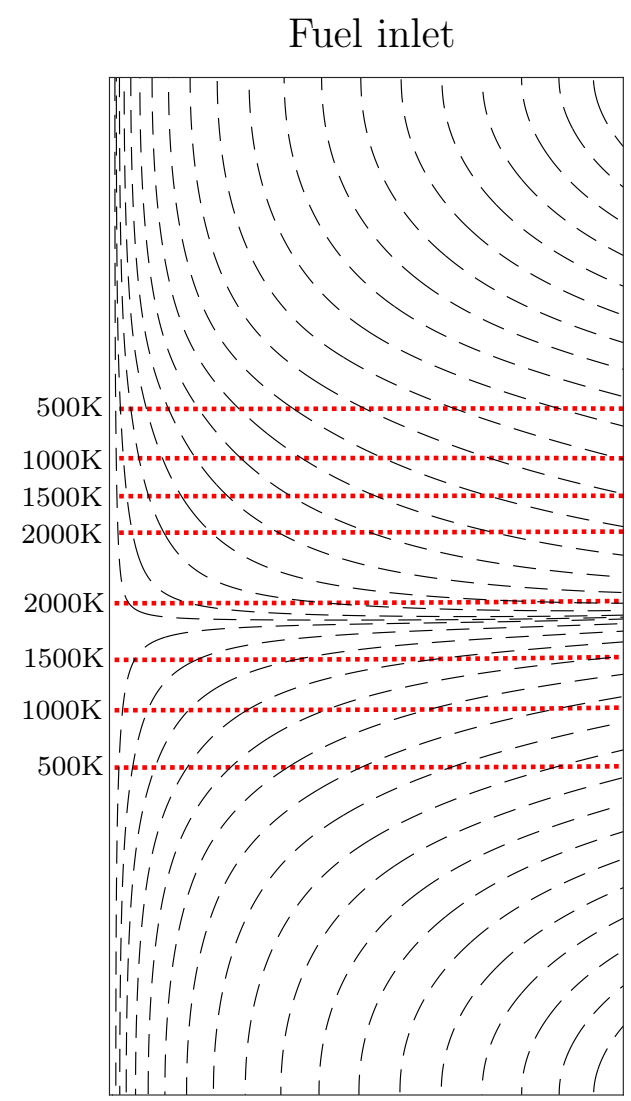

Oxidizer inlet

Figure 4: Temperature profiles in a counterflow diffusion flame (dotted lines), superimposed with streamlines (dashed lines). Fuel mixture $\left(Y_{\mathrm{C}_{3} \mathrm{H}_{8}}=0.21, Y_{\mathrm{N}_{2}}=0.79\right)$ is injected from the top nozzle, and air from the bottom. Conditions: $\rho_{f} U_{f}=\rho_{o x} U_{o x}=0.2 \mathrm{~kg} \mathrm{~m}^{2} \mathrm{~s}^{-1}$, $T_{f}=T_{o x}=300 \mathrm{~K}$, atmospheric pressure. 


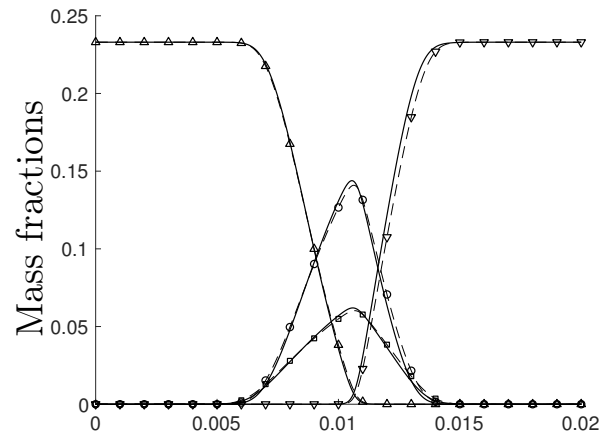

a)

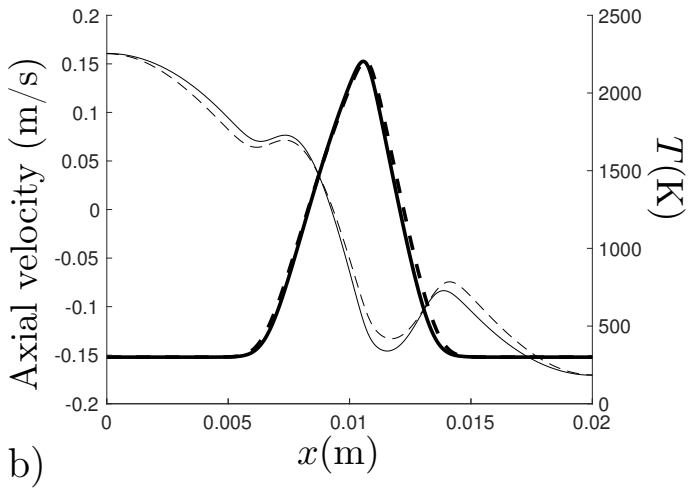

Figure 5: Counter flow diffusion flame. $\rho_{f} U_{f}=\rho_{o x} U_{o x}=0.2 \mathrm{~kg} \mathrm{~m}^{2} \mathrm{~s}^{-1}$ (a) $\mathrm{C}_{3} \mathrm{H}_{8}(\Delta)$, $\mathrm{O}_{2}(\nabla), \mathrm{CO}_{2}(\circ)$ and $\mathrm{H}_{2} \mathrm{O}(\square)$ mass fractions profiles (b). Temperature (thick lines) and axial velocity profiles, . Atmospheric pressure, temperature of fresh gases 300K. Cantera reference (plain dark line), and present model (dashed line). 


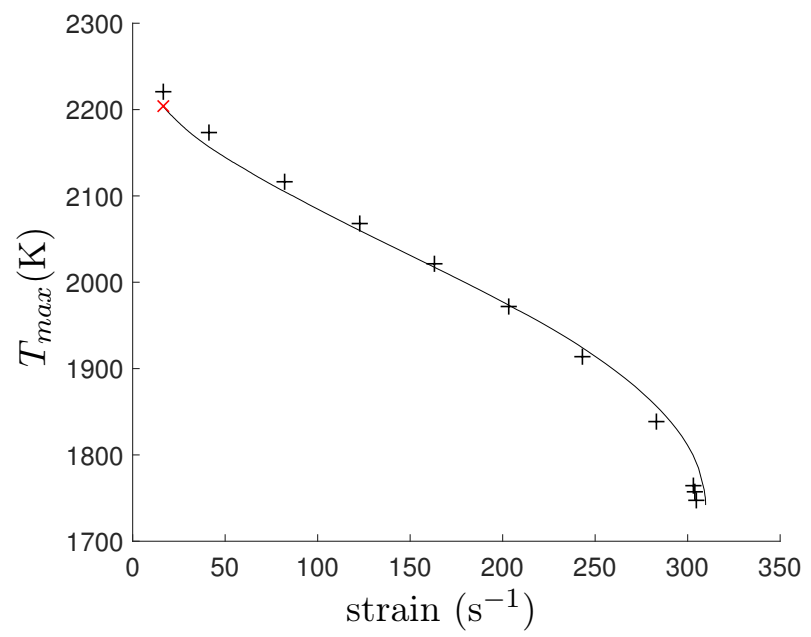

Figure 6: Maximum temperature in a counter flow diffusion flame as a function of the strain rate. Reference computed with Cantera (line), and our LB model with $L / l=100$

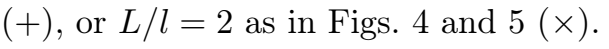

simulation domain rather than the model. The strain rate at extinction is $305 \mathrm{~s}^{-1}$ with our LB model, whereas Cantera sets it at $310 \mathrm{~s}^{-1}$. These results conclude the validation of our flow model.

\section{Concluding remarks}

A new LB combustion model has been presented. It is, to the knowledge of the authors, the first to pass the standard canonical combustion test cases of freely propagating premixed flame and counter-flow diffusion flame with strains up to extinction. In practice, the number of independent variables is the number of lattice discrete velocities (9 in our D2Q9 implementation), plus the temperature, and a $N-1$ or $N$ mass fractions ( $\mathrm{N}$ being the number of chemical species), as is typical in combustion modelling [30].

The main assumptions are:

- low Mach number 
- simplified transport properties, specified by species individual Schmidt numbers

- constant mixture heat capacity.

The last hypothesis, adopted for the sake of readability, is the most limiting in practice and is to be relaxed in our next work in preparation.

\section{Acknowledgements}

B. Cuenot, P. Sagaut and L. Vervisch are gratefully acknowledged for fruitful discussions.

We also acknowledge - for supporting Y. Feng at M2P2 - the project CLIMB, with the financial support of BPIFrance (Project No. P3543-24000) in the framework of the program "Investissement d'Avenir: Calcul Intensif et Simulation Numérique". This work was granted access to the HPC resources of Aix-Marseille Université funded by the project Equip@Meso (ANR-10EQPX-29-01), and from GENCI-TGCC/CINES (Grant 2018-A0032A07679)

[1] S. Y. Chen, G. D. Doolen, Lattice boltzmann method for fluid flows, Annual Review of Fluid Mechanics 30 (1998) 329-364.

[2] S. Marié, D. Ricot, P. Sagaut, Comparison between lattice boltzmann method and navier-stokes high order schemes for computational aeroacoustics, Journal of Computational Physics 228 (4) (2009) 1056-1070.

[3] D. Casalino, A. F. Ribeiro, E. Fares, S. Nölting, Lattice-Boltzmann aeroacoustic analysis of the lagoon landing-gear configuration, AIAA journal 52 (6) (2014) 1232-1248. 
[4] A. Sengissen, J.-C. Giret, C. Coreixas, J.-F. Boussuge, Simulations of lagoon landing-gear noise using lattice boltzmann solver, in: 21st AIAA/CEAS Aeroacoustics Conference, 2015, p. 2993.

[5] A. D'Hooge, L. Rebbeck, R. Palin, Q. Murphy, J. Gargoloff, B. Duncan, Application of real-world wind conditions for assessing aerodynamic drag for on-road range prediction, Tech. rep., SAE Technical Paper (2015).

[6] M. E. Gleason, B. Duncan, J. Walter, A. Guzman, Y.-C. Cho, Comparison of computational simulation of automotive spinning wheel flow field with full width moving belt wind tunnel results, SAE International Journal of Passenger Cars-Mechanical Systems 8 (2015-01-1556) (2015) 275-293.

[7] D. Casalino, A. Hazir, A. Mann, Turbofan broadband noise prediction using the lattice boltzmann method, AIAA Journal (2017) 1-20.

[8] M. R. Khorrami, E. Fares, B. Duda, A. Hazir, Computational evaluation of airframe noise reduction concepts at full scale, in: 22nd AIAA/CEAS Aeroacoustics Conference, 2016, p. 2711.

[9] M. R. Khorrami, E. Fares, Simulation-based airframe noise prediction of a full-scale, full aircraft, in: 22nd AIAA/CEAS aeroacoustics conference, 2016, p. 2706.

[10] O. Filippova, D. Hänel, A Novel Lattice BGK Approach for Low Mach Number Combustion, Journal of Computational Physics 158 (2) (2000) 139-160. 
[11] K. Yamamoto, X. He, G. Doolen, Simulation of combustion field with lattice Boltzmann method, Journal of statistical physics 107 (April) (2002) 367.

[12] K. Yamamoto, Combustion Simulation using the Lattice Boltzmann Method, JSME International Journal 47 (2) (2004) 4003 - 409.

[13] K. Yamamoto, N. Takada, LB simulation on soot combustion in porous media, Physica A: Statistical Mechanics and its Applications 362 (1) (2006) 111-117.

[14] S. Chen, Z. Liu, C. Zhang, Z. He, Z. Tian, B. Shi, C. Zheng, A novel coupled lattice Boltzmann model for low Mach number combustion simulation, Applied Mathematics and Computation 193 (1) (2007) 266-284.

[15] S. Chen, Z. Liu, Z. Tian, B. Shi, C. Zheng, A simple lattice Boltzmann scheme for combustion simulation, Computers and Mathematics with Applications 55 (7) (2008) 1424-1432.

[16] Z. Guo, B. Shi, N. Wang, Lattice BGK Model for Incompressible Navier - Stokes Equation, Journal of Computational Physics 165 (1) (2000) 288-306.

[17] N. I. Prasianakis, I. V. Karlin, Lattice Boltzmann method for thermal flow simulation on standard lattices, Physical Review E - Statistical, Nonlinear, and Soft Matter Physics 76 (1) (2007) 1-11.

[18] A. F. D. Rienzo, P. Asinari, E. Chiavazzo, N. I. Prasianakis, J. Mantzaras, Lattice boltzmann model for reactive flow simulations, EPL (Europhysics Letters) 98 (3) (2012) 34001. 
[19] E. Chiavazzo, I. V. Karlin, A. N. Gorban, K. Boulouchos, Coupling of the model reduction technique with the lattice Boltzmann method for combustion simulations, Combustion and Flame 157 (10) (2010) 18331849 .

[20] E. Chiavazzo, I. V. Karlin, A. N. Gorban, K. Boulouchos, Efficient simulations of detailed combustion fields via the lattice Boltzmann method, International Journal of Numerical Methods for Heat and Fluid Flow 21 (5) (2011) 494-517.

[21] C. Lin, A. Xu, G. Zhang, Y. Li, Double-distribution-function discrete boltzmann model for combustion, Combustion and Flame 164 (2016) $137-151$.

[22] Q. Li, K. Luo, Y. He, Y. Gao, W. Tao, Coupling lattice boltzmann model for simulation of thermal flows on standard lattices, Physical Review E 85 (1) (2012) 016710.

[23] Y. Feng, P. Sagaut, W. Tao, A three dimensional lattice model for thermal compressible flow on standard lattices, Journal of Computational Physics 303 (2015) 514-529.

[24] D. G. Goodwin, H. K. Moffat, R. L. Speth, Cantera: An objectoriented software toolkit for chemical kinetics, thermodynamics, and transport processes, http://www. cantera.org, version 2.3.0 (2017). doi : 10.5281/zenodo.170284.

[25] Y. Qian, D. D'Humiéres, P. Lallemand, Lattice bgk models for navierstokes equation, EPL (Europhysics Letters) 17 (1992) 479-484. 
[26] X. W. Shan, X. F. Yuan, H. D. Chen, Kinetic theory representation of hydrodynamics: a way beyond the navier-stokes equation, Journal of Fluid Mechanics 550 (2006) 413-441.

[27] P. L. Bhatnagar, E. P. Gross, M. Krook, A model for collision processes in gases. i. small amplitude processes in charged and neutral onecomponent systems, Phys. Rev. 94 (1954) 511-525.

[28] S. Chapman, T. G. Cowling, The Mathematical Theory of Non-Uniform Gases, Cambridge University Press, 1970.

[29] Z. Guo, C. Zheng, B. Shi, Discrete lattice effects on the forcing term in the lattice boltzmann method, Phys. Rev. E 65 (2002) 046308.

[30] T. Poinsot, D. Veynante, Theoretical and numerical combustion, RT Edwards, Inc., 2005.

[31] Q. Li, Y. He, Y. Wang, G. Tang, Three-dimensional non-free-parameter lattice-Boltzmann model and its application to inviscid compressible flows, Physics Letters A 373 (25) (2009) 2101 - 2108.

[32] Y. Feng, P. Sagaut, W. Q. Tao, A compressible lattice Boltzmann finite volume model for high subsonic and transonic flows on regular lattices, Computers and Fluids 131 (2016) 45-55.

[33] J. Latt, B. Chopard, Lattice boltzmann method with regularized precollision distribution functions, Mathematics and Computers in Simulation 72 (2-6) (2006) 165-168. 
[34] J. Jacob, O. Malaspinas, P. Sagaut, A new hybrid recursive regularized bhatnagar-gross-krook collision model for lattice-boltzmannmethod based large-eddy simulation, Physics of Fluids (2018) under review.

[35] S. P. Thampi, S. Ansumali, R. Adhikari, S. Succi, Isotropic discrete laplacian operators from lattice hydrodynamics, Journal of Computational Physics 234 (2013) 1-7.

[36] A. Kumar, Isotropic finite-differences, Journal of Computational Physics 201 (1) (2004) $109-118$.

[37] O. Malaspinas, B. Chopard, J. Latt, General regularized boundary condition for multi-speed lattice Boltzmann models, Computers \& Fluids 49 (1) (2011) 29-35.

[38] A. Liñán, The asymptotic structure of counterflow diffusion flames for large activation energies, Acta Astronautica 1 (7) (1974) 1007-1039.

[39] F. A. Williams, Combustion Theory, second edition., Cummings Publ. Co, 1985 .

[40] F. Shum-Kivan, Simulation des grandes echelles de flammes de spray et modélisation de la combustion non-prémélangée, Ph.D. thesis (2017). 